INPLASY

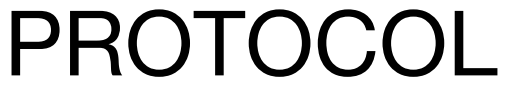

To cite: Zhou et al. Efficacy of Xianglian pill for antibioticassociated diarrhea: a protocol for a systematic review and meta-analysis. Inplasy protocol 2020100112. doi:

10.37766/inplasy2020.10.0112

Received: 28 October 2020

Published: 28 October 2020

Corresponding author:

Xin Zhou

zhouxin@cdutcm.edu.cn

Author Affiliation:

Chengdu University of

Traditional Chinese Medicine

Support: NSFC of China(No. 81703844).

Review Stage at time of this submission: The review has not yet started.

Conflicts of interest: None.

\section{Efficacy of Xianglian pill for antibiotic- associated diarrhea: a protocol for a systematic review and meta-analysis}

Zhou, X1; Zhang, X2; Gao, R³; Xiong, M" Shen, T5.

Review question / Objective: The purpose of this study is to evaluate the efficacy and safety of Xianglian pill for antibioticassociated diarrhea.

Condition being studied: Diarrhea is a common adverse effect caused by systemic antibiotic administration. The incidence of antibiotic-associated diarrhea (AAD) is in $5 \%$ to $39 \%$ of patients, from the beginning and up to two months after the end of treatment. The symptoms of AAD range from mild and self-limiting diarrhea to severe diarrhea, which caused by Clostridium difficile requires specific antibiotic treatment. Preventive measures include the use of probiotics and vaccines, but their roles are limited to the prevention of AAD. Recently, many studies have investigated that Chinese herb medicine (CHM) exerted a good curative effect for the diarrhea due to antibiotics. Xianglian pill (XLP) can treat diarrhea by shorting the duration of diarrhea and improving the absorptive function in clinical settings. However, there is currently insufficient evidence about the safety and efficacy of XLP for treating antibiotic associated diarrhea. Therefore, the purpose of this study is to systematically review current available articles to assess the effificacy and safety of the XLP treatment in patients of AAD.

INPLASY registration number: This protocol was registered with the International Platform of Registered Systematic Review and Meta-Analysis Protocols (INPLASY) on 28 October 2020 and was last updated on 28 October 2020 (registration number INPLASY2020100112).

\section{INTRODUCTION}

Review question / Objective: The purpose of this study is to evaluate the efficacy and safety of Xianglian pill for antibioticassociated diarrhea.
Rationale: The current treatment of antibiotic-associated diarrhea is not ideal. Recently, Xianglian pill is widely used in clinical practise, but there is a lack of comprehensive summary and evaluation of these studies. 
Condition being studied: Diarrhea is a common adverse effect caused by systemic antibiotic administration. The incidence of antibiotic-associated diarrhea (AAD) is in $5 \%$ to $39 \%$ of patients, from the beginning and up to two months after the end of treatment. The symptoms of AAD range from mild and self-limiting diarrhea to severe diarrhea, which caused by Clostridium difficile requires specific antibiotic treatment. Preventive measures include the use of probiotics and vaccines, but their roles are limited to the prevention of AAD. Recently, many studies have investigated that Chinese herb medicine (CHM) exerted a good curative effect for the diarrhea due to antibiotics. Xianglian pill (XLP) can treat diarrhea by shorting the duration of diarrhea and improving the absorptive function in clinical settings. However, there is currently insufficient evidence about the safety and efficacy of XLP for treating antibiotic associated diarrhea. Therefore, the purpose of this study is to systematically review current available articles to assess the effificacy and safety of the XLP treatment in patients of AAD.

\section{METHODS}

Search strategy: Sources including: 1)Cochrane Central Register of Controlled Trials (CENTRAL) on the Cochrane Library, 2) EMBASE, 3) Pubmed, 4) Chinese Biomedical Database (CBM), 5)China National Knowledge Infrastructure, 6)VIP database for Chinese technical Periodicals, and 7)Wanfang Data knowledge service platform will be systematically used to search inception without language limitation. We plan to use the following terms to search: "Chinese herbal medicine" OR "Chinese Herbal Medicine" OR "Drugs, Chinese Herbal" OR "Chinese Herbal" OR "Chinese herbal" OR "Medicine, Chinese Traditional" OR "Traditional Chinese Medicine" OR "traditional Chinese medicine" OR "Traditional Chinese medicine decoction") AND ("diarrhea" OR "diarrhe" OR "diarrhoe" OR "diarhe" OR "diarhoe"OR "dysentery" OR “dysenter" OR "gastroenteritis" OR "gastroenteritis" OR "gastro enteritis"). The work will be independently conducted by 2 authors (Xin Zhou and Xiaobo Zhang).

Participant or population: Participants (age 6 months to 80 years) were men and women who underwent antibiotic therapy for any reason as outpatients or inpatients.

Intervention: Both groups were cured with conventional treatments recommended by the guidelines. Interventions in the experimental group are Xianglian pill (any formulation, any dose, any duration) combined with conventional medicine. Modified Xianglian pill will also be included as long as it contains the 2 herbs and increases not exceeding 10 herbs. The interventions in the control group are routine drug therapy in guidelines. All the formulas involved should keep to the principles of Monarch, Minister, Assistant, and Guide in traditional Chinese medicine prescription. Moreover, herbal food, nutritional supplements or no intervention will be excluded.

Comparator: The control group that receives routine medication alone, health education, placebo, non-traditional Chinese active agents or no treatment will be eligible for inclusion in the review.

Study designs to be included: Randomized controlled trials (RCTs) that reported the effect of Xianglian pill for the treatment of different degrees of diarrhea caused by antibiotic administration will be included for this study.

Eligibility criteria: Studies will be selected according to the PICOS criteria outlined in the referred ssections.

Information sources: We plan to search the following databases: Cochrane Central Register of Controlled Trials (CENTRAL) on the Cochrane Library, EMBASE, Pubmed, Chinese Biomedical Database (CBM), China National Knowledge Infrastructure, VIP database for Chinese technical Periodicals, and Wanfang Data knowledge service platform. 
Main outcome(s): The main outcomes include effectiveness, remarkable result, recovery condition, improvement, remission rate, relapse rate, adverse events, clinical absolute score, and relative score.

Additional outcome(s): The secondary outcomes include mean duration of diarrhea and microbiome characteristics.

Data management: Records from database searches will be processed by EndNoteX8 software. The information, including author, year of publication, sample size, intervention, and outcome will be extracted from each study and entered into the spreadsheet. Two review authors will be responsible for information searching and checking from each included trial independently according to the protocol. Any disagreement will be resolved by discussion until consensus is reached or by consulting a third review author. A data spreadsheet will be created using Microsoft Excel 2010 to collect relevant information and data.The following data will be extracted: author, publication time, article title, country where the study is conducted, language, study setting, study period, total number of people included in the study, funding source, definition and diagnostic criteria for diarrhea, inclusion and exclusion criteria for participants, patient characteristics (age, gender, diagnosis, socioeconomic status), number of patients allocated to each group, presence/absence of intention and efficacy data to treat analysis. For some outcomes, if the author only provide figure, then we will request the data from authors or extract the data from the figure using software. For articles published in abstract form only, we will contacted corresponding authors of the included trials with incomplete data, including unclear risk of bias ratings, for additional information. Two review authors will independently extract information from each included trial according to pre-specified selection criteria. Any disagreement will be resolved by discussion until consensus is reached or by consulting a third review author.
Endnote and excel software will be used for data extraction.

Quality assessment / Risk of bias analysis: Quality components for each included RCT will be assessed for selection, detection, performance, reporting and loss to followup bias. Each of the included studies will be independently evaluated by two authors according to the Cochrane Collaboration's tool for randomized controlled trials. Items will be evaluated in three categories: Low risk of bias, unclear bias and high risk of bias. The domains of evaluation include sequence generation, allocation concealment, blinding, incomplete outcome data, selective outcome reporting, and other sources of bias. Disagreement will be resolved by discussion or the third party. Bias risk assess figure will be drawn by RevMan5.3 software.

Strategy of data synthesis: The data will be analyzed using RevMan5.3 software. Risk ratio (RR) for both fixed and random effects models (weighting by inverse of variance) will be used between the experimental and control groups with $95 \%$ confidence intervals (Cls). Between-study zero heterogeneity will be assessed using the $\mathbf{T}^{2}, \mathrm{X}^{2}$ (Cochran $Q$ ) and $I^{2}$ statistics. According to the Cochrane handbook, the 12 will be considered non-important $(60 \%)$. Results will be assessed using forest plots and presented as RRs for the main outcome and secondary outcomes. An influence analysis will be performed to ascertain the results of the meta-analysis by excluding each of the individual studies. Publication bias will be assessed by a funnel plot for meta-analysis and quantified by the Egger method. Statistical analysis will be conducted using STATA software for Mac v15.0 (Stata Corp., College Station, Texas) and $R$ studio v1.0.136 (The $R$ Foundation for Statistical Computing).

Subgroup analysis: To explore possible explanations for heterogeneity, subgroup analyses will be performed according to the following items: duration or severity of antibiotic-associated diarrhea, children or 
adults, the original or Modified of Xianglian pill, duration or dosage of herbal medicine treatment, and the type of treatment in the control group.

Sensibility analysis: When authors neglect to report PICO related items of interest, we will contact them via email. To assess the potential influence of missing outcome responses (e.g. children lost to follow-up). Sensitivity analyses will be applied for the primary outcomes, incidence of diarrhea and adverse events. Although many approaches exist for evaluating the sensitivity of results for missing outcome data, we will elect to make assumptions about the missing data which were extreme but still plausible. Sensitivity analyses will be conducted using a fixed-effect model as compared to random-effects, and we will assess the potential influence of missing participant outcome data as compared to a complete case analysis, with the method described above.

Language: No restrictions.

Country(ies) involved: China.

Other relevant information: None.

Keywords: antibiotic-associated diarrhea, Xianglian pill, Randomized controlled trial, Systematic review, Meta-analysis.

Dissemination plans: After successful registration of this protocol, it is expected that data collection and Review (that is, official articles) will be completed within 12-18 months. Two review authors (Xin Zhou and Xiaobo Zhang) will be responsible for information searching and extraction from each included trial independently according to the protocol. Any disagreement will be resolved by discussion until consensus is reached or by consulting a third review author (Rui Gao). Xin Zhou will draft the manuscript, and Tao Shen is the study guarantor.

Contributions of each author:

Author 1 - Xin Zhou.

Author 2 - Xiaobo Zhang.

Author 3 - Rui Gao.
Author 4 - Min Xiong.

Author 5 - Tao Shen. 